\title{
COVID-19 Clinical Trials Registered Worldwide for Drug Intervention: An Overview and Characteristic Analysis
}

This article was published in the following Dove Press journal: Drug Design, Development and Therapy

\author{
Bin Wang (D) \\ Junkai Lai' \\ Xiaoyan Yan $^{2}$ \\ Feifei Jin' \\ Bin $\mathrm{Yi}^{3}$ \\ Caixia $\mathrm{An}^{3}$ \\ Yuanxiao $\mathrm{Li}^{4}$ \\ Chen Yaol,2 \\ 'Department of Biostatistics, Peking \\ University First Hospital, Beijing, People's \\ Republic of China; ${ }^{2}$ Peking University \\ Clinical Research Institute, Beijing, \\ People's Republic of China; ${ }^{3}$ Children's \\ Emergency Center, Gansu Provincial \\ Maternity and Child-Care Hospital, \\ Lanzhou, People's Republic of China; \\ ${ }^{4}$ Department of Pediatric \\ Gastroenterology, Second Hospital of \\ Lanzhou University, Lanzhou, People's \\ Republic of China
}

Correspondence: Chen Yao

Peking University First Hospital, Xicheng District, Beijing 100034, People's Republic of China

Tel +8618610640562

Email yaochen@hsc.pku.edu.cn
Objective: This study aims to comprehensively evaluate the characteristics of clinical drug trials to facilitate the collection of evidence for COVID-19 drug treatments.

Methods: A retrospective analysis of 910 trials retrieved on August 7, 2020.

Results: A total of 910 registered clinical trials with at least one drug intervention were evaluated. The number of registrations $(32.4 \%, 295)$ from the United States accounted for nearly one-third of the total and far exceeded that of other countries individually. Furthermore, the peak number of trials were registered in April $(34.3 \%, 312)$. Over half of the trials $(51.2 \%, 466)$ are in the recruitment phase, and only $4.2 \%$ (38) of the trials have been completed. The median (interquartile range) estimated enrollment is $127(59,365)$. In $39 \%$ (355) of trials, the estimated enrollment is less than 100 participants. A total of $94.5 \%$ (790) of the trials use randomization in the allocation, $82.7 \%$ (753) use a parallel intervention mode, and 52.2\% (475) use masking. A total of 287 drug names have been standardized and mapped. "Hydroxychloroquine" is the leading drug among the registered trials $(7.47 \%, 68)$. Among the main countries contributing to investigations on "hydroxychloroquine", the United States ranks first with $36.76 \%$ (25) of the trials.

Conclusion: The designs of COVID-19 clinical drug trials have greatly improved in terms of the implementation of randomization and, particularly, blinding methods. In terms of drug reuse, the number of drug types has greatly increased, and hundreds of drugs have been used for efficacy screening. The emergence of large-sample registration trials is expected to address the uncertainty regarding the current clinical efficacy of some drugs.

Keywords: COVID-19, clinical trials, investigation, intervention, drug, treatment

\section{Introduction}

Since December 2019, the outbreak of coronavirus disease 2019 (COVID-19) has been disastrous for everyone. ${ }^{1}$ Given the global COVID-19 pandemic, the search for an effective treatment drug has become a common goal for researchers worldwide. Although various potential drugs have been explored since the start of the epidemic, no drugs with significant efficacy have been found through existing large-scale COVID-19 clinical trials. ${ }^{2}$ In the face of this urgent epidemic, some researchers mistakenly believe that large randomized controlled trials are not applicable or ethical. Due to this misunderstanding, previous studies have suggested that COVID-19 clinical trials registered before March 2020 have many study design defects, such as a lack of blinding. ${ }^{3}$ As the epidemic has spread throughout the world and a total of 3000 clinical research studies have been registered on the American Clinical Trials Registry 
(ClinialTrials.gov), an evaluation of the characteristics of studies investigating registered drug interventions is needed. $^{2}$ Therefore, the purpose of this study is to comprehensively evaluate the characteristics of clinical trials with at least one drug intervention registered on ClinialTrials.gov to facilitate the collection of evidence for the efficacy of COVID-19 drug treatments.

\section{Methods}

On August 7, 2020, we retrieved data from the ClinialTrials.gov. The MeSH Supplementary Concept of "COVID-19" was used as a search keyword. The entire retrieval and webpage data extraction process was completed independently by two authors, and finally, the results were compared.

\section{Selection Criteria}

The selection criteria for registered trials included (1) COVID-19 studies with at least 1 drug intervention type; (2) Interventional studies (clinical trial); and (3) Studies whose first-registration research goals were to performing research on COVID-19 and not updating previous research to include COVID-19.

\section{Data Summarization and Visualization}

Python (version 3.7.7) was used to perform all tasks, including data extraction and statistical analysis. To visualize the registrations of drug intervention clinical trials worldwide, the world map was drawn using the "plotly" package (version 4.8.2). One way to visualize the relationship between different countries and drugs is to draw a Sankey diagram using the "holoviews" package (version 1.13.3). We selected a few drugs with the largest number of studies and showed the main countries that contributed to investigating these drugs. To avoid generating an excessively complex Sankey diagram and increase readability, we controlled the number of overall nodes to be able to label the interactions between drugs and countries. The Sankey diagram was used to display the flow of the number of trials from one country to a drug as arrows, in which the width of the arrows was proportional to the degree of flow.

\section{Results}

\section{Search Results}

Prior to August 7, 2020, a total of 2928 COVID-19 studies were registered on ClinialTrials.gov. A summary of these studies is shown in Table 1. We implemented strict study screening according to the inclusion criteria. The flow diagram of the study screening is shown in Figure 1. A total of 910 clinical trial registrations with at least one drug intervention were ultimately included.

\section{Global Distribution of the Number of Registered Trials}

The included trials involved a total of 62 countries. The United States was the leading country in terms of the total number of registered trials $(32.4 \%, 295)$, followed by China $(6.5 \%, 59)$, France $(6.4 \%, 58)$, Spain $(5.4 \%, 49)$, and the United Kingdom $(4.7 \%, 43)$. The number of registered trials among the top 5 countries accounted for $55.4 \%$ of all included trials. The detailed global distribution of the number of included trials is shown in Figure 2.

\section{Trends in Trial Registration}

Figure 3 presents the number of new trials with at least one drug intervention registered in ClinialTrials.gov each month and by country. Based on the number of trials, the top five countries are listed, and data from the remaining countries are combined. It can be seen from the line chart that the peak of trial registration occurred in April (34.3\%, 312). The earliest registration came from a trial from China registered in January. As a result of the timing of the outbreak, China hit a peak in its trial registration in February, earlier than any other country. It should be noted that the August numbers are relatively small because the data were collected in early August.

\section{General Characteristics of the Registered Trials}

We summarized the general characteristics of the included trials in Table 2. Since the proportion of trials from the United States $(32.4 \%, 295)$ is close to one-third, we grouped the United States and other countries separately to better show

Table I Summary of the Registered COVID-19 Studies

\begin{tabular}{|l|l|l|l|}
\hline Selected Search of ClinialTrials.gov & Number of Studies & Number of Countries & Number of US States and Washington, D.C. \\
\hline All COVID-19 Studies & 2928 & 112 & 51 \\
At Least One US Location & 631 & 67 & 51 \\
US Federal Funding & 71 & 23 & 50 \\
\hline
\end{tabular}



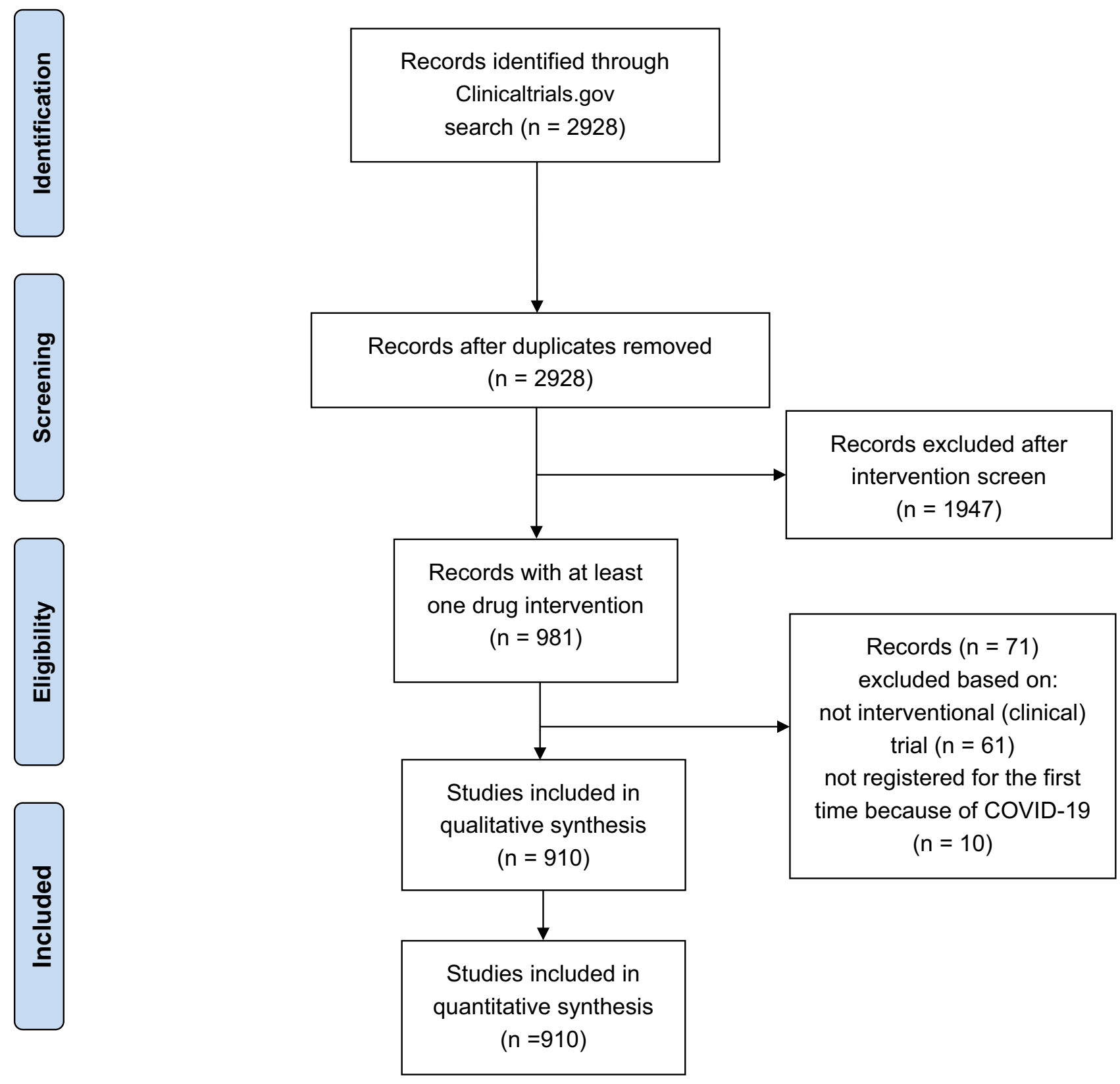

Figure I Study selection flow diagram.

the distribution of the characteristics. Half of all the trials $(51.2 \%, 466)$ are in the recruitment phase, and $4.2 \%$ (38) of the trials have been completed; therefore, a large portion of the trials had not yet started recruiting participants. The age of the patients included in most trials $(87.6 \%, 797)$ is in the "adult, older adult" category (generally 18 years and older). Most trials are in Phase 2 (39.5\%, 359). The median (interquartile range) estimated enrollment is $127(59,365)$. In $39 \%$ (355) of the trials, the estimated enrollment is less than 100 participants. There are $30.8 \%$ (280) trials with an estimated enrollment of 300 participants and above. A total of $94.5 \%$ (790) of the trials use random allocation, $82.7 \%$ (753) employ a parallel intervention mode, and 52.2\% (475) use masking. The primary purpose of $86.3 \%$ (785) of the trials is treatment. The primary outcome indicators were categorized and displayed as bar graphs (Figure 4). The use ratio of the well-recognized sevencategory ordinal scale (time to clinical improvement) ranks second, closely following viral load.

\section{Trial Drugs}

Standardized drug names can be obtained from ClinialTrials. gov. A total of 287 drug names were standardized and mapped. "Hydroxychloroquine" is the leading drug among the registered trials $(7.47 \%, 68)$, followed by "azithromycin, 


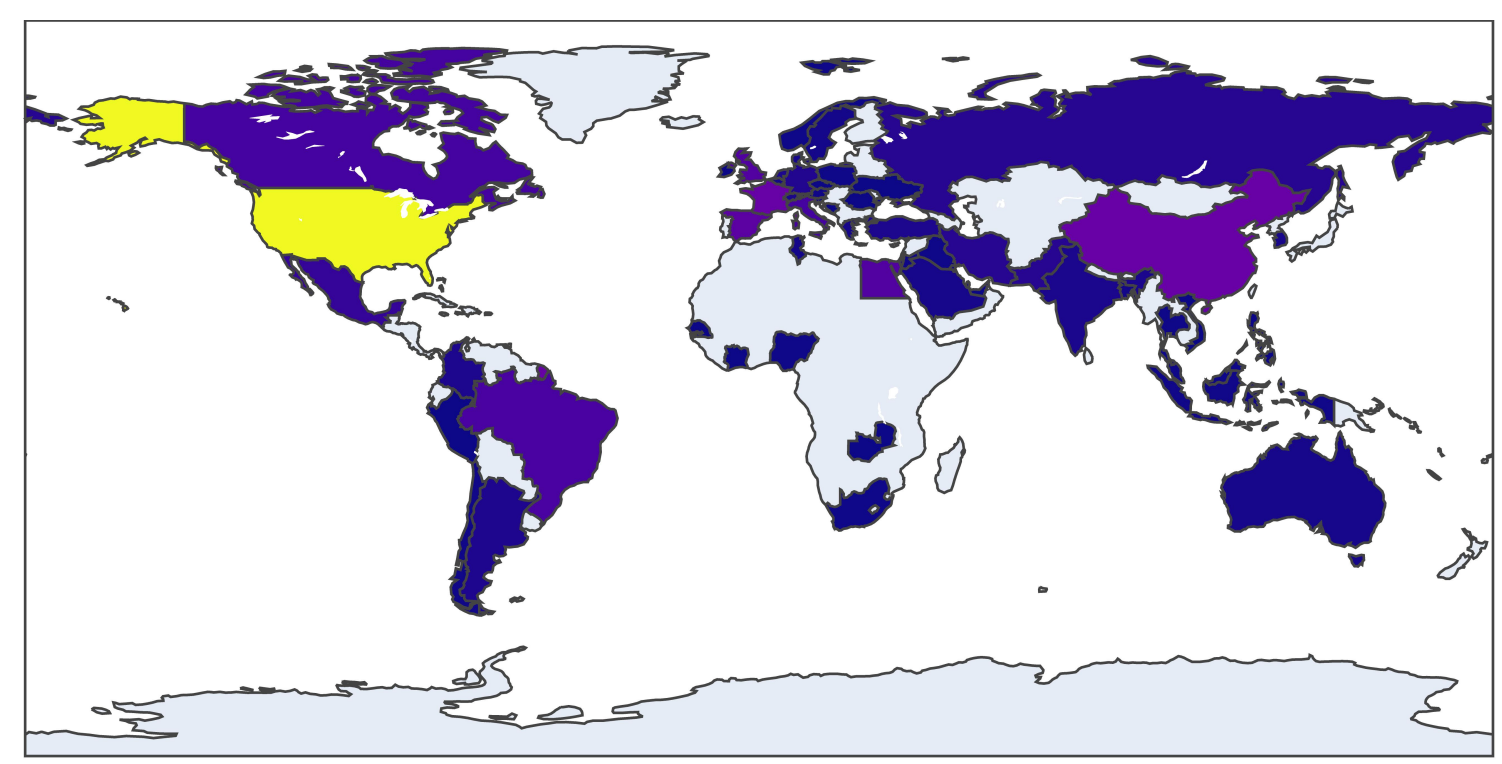

Number

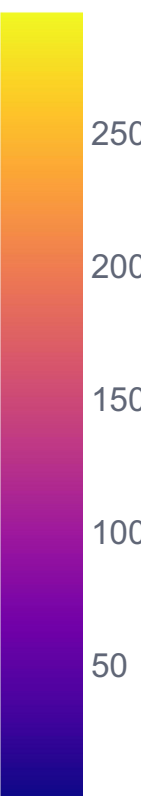

Figure 2 Number of COVID-19 clinical trial registrations with at least one drug intervention in each area.

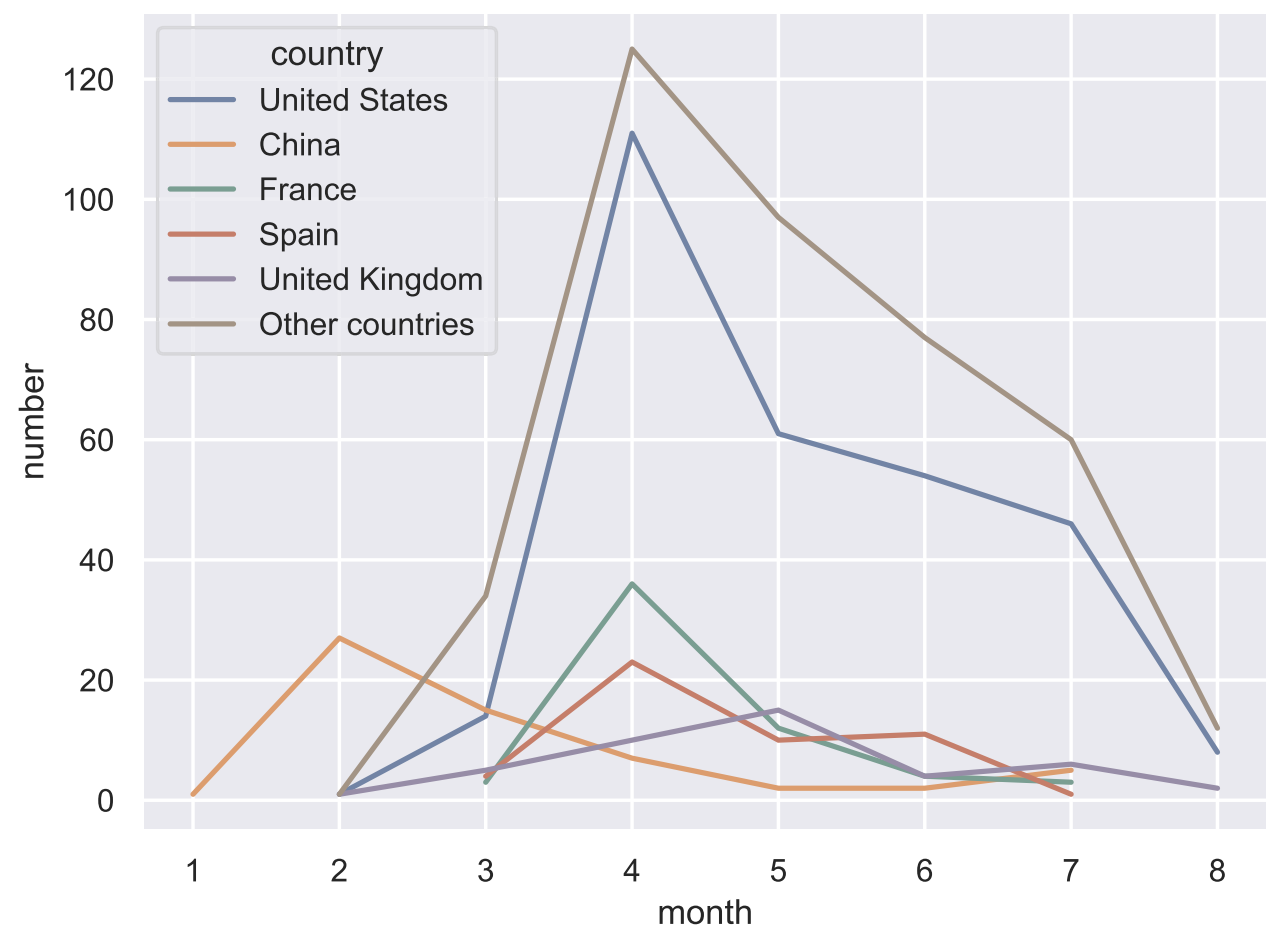

Figure 3 Number of new trials with at least one drug intervention registered in ClinialTrials.gov each month and by country. Based on the number of trials, the top five countries are listed, and data from the other countries are combined.

hydroxychloroquine" $(2.97 \%, 27)$, "tocilizumab" $(1.98 \%$, $18)$, "favipiravir" $(1.76 \%, 16)$, and "ruxolitinib" $(1.21 \%$, 11). The number of registered trials investigating the top 5 drugs accounted for a quarter (15.39\%) of all included trials. The top 30 drugs are shown in Figure 5.

\section{Relationship Between Country and Drug}

Since drug trials may be distributed across multiple countries, we used a Sankey diagram to visualize the drugs of interest and its main countries that contributed to their investigation. We first selected the top 9 drug nodes based 
Table 2 General Characteristics of COVID-19 Clinical Trial Registrations with at Least One Drug Intervention

\begin{tabular}{|c|c|c|c|c|c|}
\hline Item & Category & Missing & Overall $(n=910)$ & United States $(n=295)$ & Other Countries $(n=615)$ \\
\hline Status, n (\%) & $\begin{array}{l}\text { Active, not recruiting } \\
\text { Completed } \\
\text { Enrolling by invitation } \\
\text { Not yet recruiting } \\
\text { Recruiting } \\
\text { Suspended } \\
\text { Terminated } \\
\text { Withdrawn }\end{array}$ & 0 & $\begin{array}{l}40(4.4) \\
38(4.2) \\
18(2.0) \\
310(34.1) \\
466(51.2) \\
15(1.6) \\
8(0.9) \\
15(1.6)\end{array}$ & $\begin{array}{l}13(4.4) \\
2(0.7) \\
11(3.7) \\
90(30.5) \\
167(56.6) \\
5(1.7) \\
1(0.3) \\
6(2.0)\end{array}$ & $\begin{array}{l}27(4.4) \\
36(5.9) \\
7(1.1) \\
220(35.8) \\
299(48.6) \\
10(1.6) \\
7(1.1) \\
9(1.5)\end{array}$ \\
\hline Age, n (\%) & $\begin{array}{l}\text { Adult } \\
\text { Adult/Older Adult } \\
\text { Child } \\
\text { Child/Adult } \\
\text { Child/Adult/Older Adult } \\
\text { Older Adult }\end{array}$ & 0 & $\begin{array}{l}37(4.1) \\
797(87.6) \\
1(0.1) \\
3(0.3) \\
62(6.8) \\
10(1.1)\end{array}$ & $\begin{array}{l}\text { I (0.3) } \\
275(93.2) \\
- \\
2(0.7) \\
14(4.7) \\
3(1.0)\end{array}$ & $\begin{array}{l}36(5.9) \\
522(84.9) \\
I(0.2) \\
I(0.2) \\
48(7.8) \\
7(1.1)\end{array}$ \\
\hline Sex, n (\%) & $\begin{array}{l}\text { All } \\
\text { Female } \\
\text { Male }\end{array}$ & 0 & $\begin{array}{l}895(98.4) \\
4(0.4) \\
11(1.2)\end{array}$ & $\begin{array}{l}292(99.0) \\
I(0.3) \\
2(0.7)\end{array}$ & $\begin{array}{l}603(98.0) \\
3(0.5) \\
9(1.5)\end{array}$ \\
\hline Phases, n (\%) & $\begin{array}{l}\text { Early Phase I } \\
\text { Not Applicable } \\
\text { Phase I } \\
\text { Phase I/Phase } 2 \\
\text { Phase 2 } \\
\text { Phase 2/Phase } 3 \\
\text { Phase } 3 \\
\text { Phase } 4\end{array}$ & 0 & $\begin{array}{l}15(1.6) \\
54(5.9) \\
51(5.6) \\
45(4.9) \\
359(39.5) \\
106(11.6) \\
211(23.2) \\
69(7.6)\end{array}$ & $\begin{array}{l}5(1.7) \\
2(0.7) \\
22(7.5) \\
20(6.8) \\
169(57.3) \\
17(5.8) \\
43(14.6) \\
17(5.8)\end{array}$ & $\begin{array}{l}10(1.6) \\
52(8.5) \\
29(4.7) \\
25(4.1) \\
190(30.9) \\
89(14.5) \\
168(27.3) \\
52(8.5)\end{array}$ \\
\hline Enrollment, median (QI, Q3) & & 0 & $127(59,365)$ & $100(45,300)$ & $144(60,387)$ \\
\hline Enrollment classification, n (\%) & $\begin{array}{l}<50 \\
50 \sim 99 \\
100 \sim 199 \\
200 \sim 299 \\
300 \sim 499 \\
500 \sim 999 \\
1000 \sim 9999 \\
\geq 10,000\end{array}$ & 0 & $\begin{array}{l}195(21.4) \\
160(17.6) \\
171(18.8) \\
104(11.4) \\
115(12.6) \\
77(8.5) \\
81(8.9) \\
7(0.8)\end{array}$ & $\begin{array}{l}82(27.8) \\
54(18.3) \\
50(16.9) \\
27(9.2) \\
31(10.5) \\
26(8.8) \\
23(7.8) \\
2(0.7)\end{array}$ & $\begin{array}{l}113(18.4) \\
106(17.2) \\
121(19.7) \\
77(12.5) \\
84(13.7) \\
51(8.3) \\
58(9.4) \\
5(0.8)\end{array}$ \\
\hline Funded By, n (\%) & $\begin{array}{l}\text { Industry } \\
\text { Industry/NIH/Other } \\
\text { Industry/Other } \\
\text { Industry/Other/NIH } \\
\mathrm{NIH} \\
\mathrm{NIH} / \text { Other } \\
\mathrm{NIH} / \mathrm{O} \text { ther/US Fed } \\
\text { Other } \\
\text { Other/Industry } \\
\text { Other/NIH } \\
\text { Other/NIH/Industry } \\
\text { US Fed } \\
\text { US Fed/Other }\end{array}$ & 0 & $\begin{array}{l}156(17.1) \\
1(0.1) \\
53(5.8) \\
1(0.1) \\
3(0.3) \\
1(0.1) \\
1(0.1) \\
616(67.7) \\
67(7.4) \\
6(0.7) \\
2(0.2) \\
2(0.2) \\
1(0.1)\end{array}$ & $\begin{array}{l}77(26.1) \\
\text { I }(0.3) \\
\text { I8 (6.I) } \\
\text { I }(0.3) \\
\text { I }(0.3) \\
\text { I }(0.3) \\
\text { I }(0.3) \\
\text { I58 (53.6) } \\
27(9.2) \\
5(1.7) \\
2(0.7) \\
2(0.7) \\
\text { I }(0.3)\end{array}$ & $\begin{array}{l}79(12.8) \\
- \\
35(5.7) \\
- \\
2(0.3) \\
- \\
- \\
458(74.5) \\
40(6.5) \\
I(0.2) \\
- \\
- \\
-\end{array}$ \\
\hline
\end{tabular}

(Continued) 
Table 2 (Continued).

\begin{tabular}{|c|c|c|c|c|c|}
\hline Item & Category & Missing & Overall $(n=910)$ & United States $(n=295)$ & Other Countries $(n=6 \mid 5)$ \\
\hline Allocation, n (\%) & $\begin{array}{l}\text { Nonrandomized } \\
\text { Randomized }\end{array}$ & 74 & $\begin{array}{l}46(5.5) \\
790(94.5)\end{array}$ & $\begin{array}{l}17(6.4) \\
248(93.6)\end{array}$ & $\begin{array}{l}29(5.1) \\
542(94.9)\end{array}$ \\
\hline Intervention Model, n (\%) & $\begin{array}{l}\text { Crossover } \\
\text { Factorial } \\
\text { Parallel } \\
\text { Sequential } \\
\text { Single }\end{array}$ & 0 & $\begin{array}{l}18(2.0) \\
21(2.3) \\
753(82.7) \\
30(3.3) \\
88(9.7)\end{array}$ & $\begin{array}{l}3(1.0) \\
9(3.1) \\
233(79.0) \\
12(4.1) \\
38(12.9)\end{array}$ & $\begin{array}{l}15(2.4) \\
12(2.0) \\
520(84.6) \\
18(2.9) \\
50(8.1)\end{array}$ \\
\hline Masking, n (\%) & $\begin{array}{l}\text { Double } \\
\text { None } \\
\text { Quadruple } \\
\text { Single } \\
\text { Triple }\end{array}$ & 0 & $\begin{array}{l}127(14.0) \\
435(47.8) \\
186(20.4) \\
79(8.7) \\
83(9.1)\end{array}$ & $\begin{array}{l}54(18.3) \\
106(35.9) \\
81(27.5) \\
14(4.7) \\
40(13.6)\end{array}$ & $\begin{array}{l}73(11.9) \\
329(53.5) \\
105(17.1) \\
65(10.6) \\
43(7.0)\end{array}$ \\
\hline Primary Purpose, n (\%) & $\begin{array}{l}\text { Basic Science } \\
\text { Diagnostic } \\
\text { Health Services Research } \\
\text { Other } \\
\text { Prevention } \\
\text { Supportive Care } \\
\text { Treatment }\end{array}$ & 0 & $\begin{array}{l}\text { I }(0.1) \\
\text { I }(0.1) \\
\text { I }(0.1) \\
7(0.8) \\
106(11.6) \\
9(1.0) \\
785(86.3)\end{array}$ & $\begin{array}{l}\text { I }(0.3) \\
\text { I }(0.3) \\
- \\
- \\
38(12.9) \\
3(1.0) \\
252(85.4)\end{array}$ & $\begin{array}{l}- \\
- \\
I(0.2) \\
7(1.1) \\
68(11.1) \\
6(1.0) \\
533(86.7)\end{array}$ \\
\hline
\end{tabular}

on the number of trials investigating them (with counts greater than 9) and then filtered out the main countries contributing to the investigation of these drugs (a total of 8 national nodes with counts greater than 6). The filtering threshold was manually determined according to the line density of the Sankey diagram. Among the main countries contributing to the investigation of "hydroxychloroquine", the United States registered 36.76\% (25) of the trials, and Canada registered $10.29 \%$ (7). For "azithromycin, hydroxychloroquine", $33.33 \%$ (9) of the trials were from the United States, and $22.22 \%$ (6) of the trials were from France. For "tocilizumab", 33.33\% (6) of trials were from the United States, and $16.67 \%$ (3) of trials were from Italy. Other detailed relationships are shown in Figure 6.

\section{Discussion}

Since the outbreak of the COVID-19 pandemic, clinical trials of therapeutic drugs for the treatment of this disease have become a focus of attention from researchers. The dramatic increase in the number of clinical trials has caused concern over their quality and level of evidence in the treatment of COVID-19. For example, when the outbreak first appeared in China, the clinical studies registered by some researchers on the Chinese Clinical Trial Registry (http://www.chictr.org.cn) had many design quality problems, such as a lack of blinding, replicated research, small sample sizes, and unreasonable main outcome indicators. ${ }^{3}$ Although retractions of clinical studies receive proper attention from researchers, ${ }^{4}$ this has been amplified for clinical studies on COVID-19., ${ }^{5,6}$ In addition, there is persistent controversy regarding the results of some COVID-19 drug trials, such as those investigating angiotensin-converting enzyme inhibitors/angiotensin-receptor blockers (ACEIs/ARBs), ${ }^{7}$ hydroxychloroquine, ${ }^{8}$ and chloroquine. ${ }^{9}$ These phenomena necessitate the further evaluation of registered COVID-19 trials.

This is the first overview and characteristic analysis of COVID-19 clinical trial registrations with at least 1 drug intervention. A previous study only retrieved 24 drug clinical trials from ClinialTrials.gov on March 12, $2020 ;{ }^{10}$ since then, the number of trials has increased 38 fold (910). From the global distribution map of registered trials of COVID-19 drugs, the number of registrations from the United States accounts for nearly one-third of the total and far exceeds that of other countries. The large number of COVID-19 patients in the United States is an important factor in its large percentage of clinical trial registrations. Therefore, in Table 2, a separate column for the United States in terms of trial characteristics is shown to the reader if interested. Currently, randomized, double- 


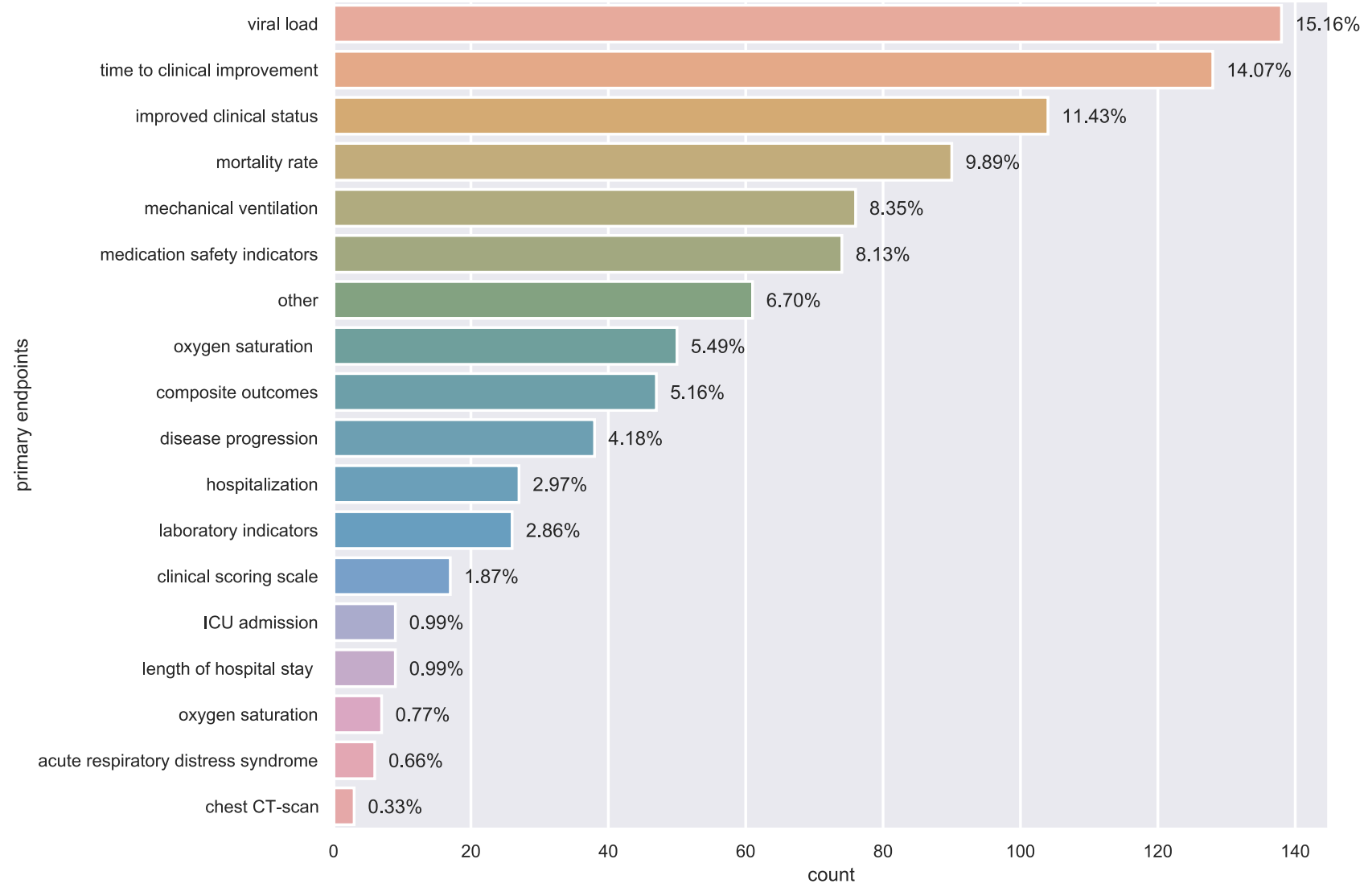

Figure 4 Number of registered trials by primary outcome indicator.

blind and controlled conditions remain the gold standard for evaluating the quality of COVID-19 clinical trial designs. Given the early outbreak of the epidemic in China and due to the urgency of the event, many studies did not include blinding. The global spread of the epidemic has given researchers more time to prepare their studies. Compared with previous studies, ${ }^{3}$ our results show that the proportion of randomization increased from $71 \%$ to $94.5 \%$, while the blinding rate increased from $5 \%$ to $52.2 \%$. The increase in these numbers reflects the importance global researchers now place on the design quality of clinical drug trial for COVID-19. Another phenomenon we observed is the increase in trials with large sample sizes. According to the results of our survey, the number of trials with more than 500 samples reached $15.5 \%$ (165), which can provide higher-quality evidence for drug treatment. Nevertheless, more than one-third of the trials $(39 \%$, 355) were small-sample (less than 100 participants) studies that may not provide reliable conclusions.

Some previous studies evaluated the research characteristics of COVID-19 clinical trials. ${ }^{11-15}$ A similar study analyzing COVID-19 clinical trials had a search time very close to ours, and they outline the results of some suitable drugs. ${ }^{12}$ Therefore, we will focus on some of the latest research results for these drugs. Judging from the current ranking of drug popularity, the percentage of trials investigating "hydroxychloroquine" reached $7.47 \%$ (68), ranking first. In the past few months, chloroquine and hydroxychloroquine were approved by the FDA for testing as a treatment for COVID-19. ${ }^{11,12}$ Hydroxychloroquine is a chemically modified chloroquine derivative with a similar mechanism of action as chloroquine but better absorption and fewer side effects. ${ }^{12}$ It can be seen from the "hydroxychloroquine" node connections in the Sankey diagram that the United States is the main contributor $(36.76 \%, 25)$ to "hydroxychloroquine" investigations. A multinational analysis of the largest sample of hydroxychloroquine trials indicated that the drug may cause severe arrhythmia and even sudden cardiac death in patients with COVID-19. After the preliminary results were announced in the Randomised Evaluation of COVID-19 Therapy (RECOVERY Trial or RECOVERY), both the National Institutes of Health (NIH) and World Health Organization (WHO) suspended the COVID-19 


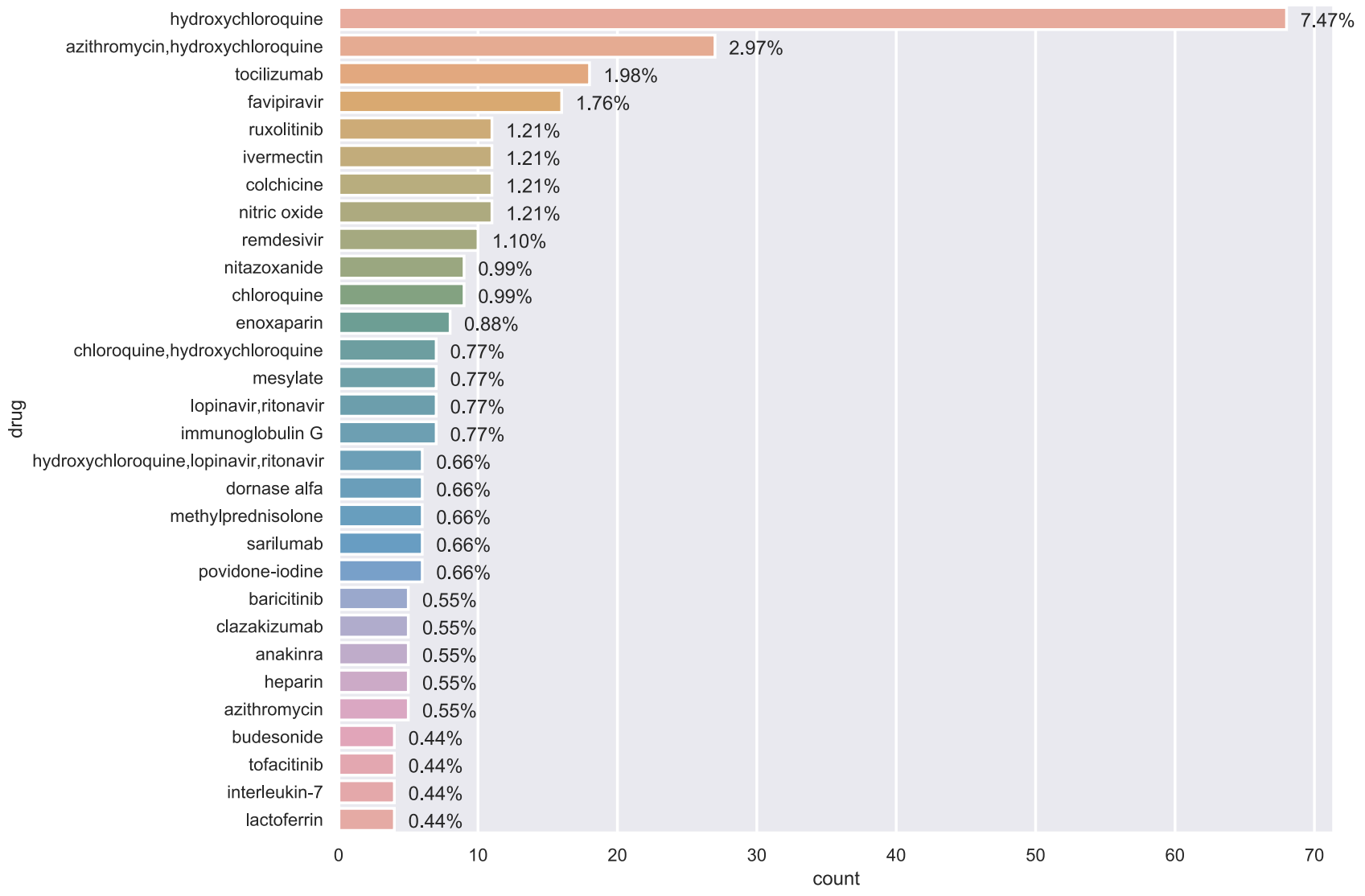

Figure 5 Number of registered trials by mapped drug name. Only the top 30 drugs are shown. The percentage on the bar graph indicates the proportion of trials for the drug out of the total trials. Note: if a trial contains multiple drugs, it will be counted once for each of the drugs. Standardized drug names automatically assigned by the National Library of Medicine based on drug names provided by the responsible party.

hydroxychloroquine trial given its lack of benefits. A network meta-analysis of COVID-19 drug treatments showed that hydroxychloroquine may not reduce mortality (low certainty) or the need for mechanical ventilation (moderate certainty). ${ }^{16}$ In addition, the present review indicates that hydroxychloroquine has the potential to cause major harm, but the extent to which it causes adverse reactions cannot be determined. The results of the recently published RECOVERY trial on hydroxychloroquine $^{17}$ among hospitalized COVID-19 patients are similar to the interim results (published in the medRxiv) of the WHO Solidarity trial, ${ }^{18}$ which concluded that hydroxychloroquine cannot reduce the mortality of inpatients. These findings indicate that hydroxychloroquine is not an effective treatment for COVID-19 patients. Another very influential trial evaluated hydroxychloroquine as a postexposure prophylaxis and yielded a negative result. ${ }^{19}$

As one of the largest international randomized trials, the Solidarity trial recruited nearly 12,000 patients across 500 hospitals in more than 30 countries. It provided conclusive evidence on the effectiveness of 4 repurposed drugs (remdesivir, hydroxychloroquine, lopinavir/ritonavir and interferon). For overall mortality, initiation of ventilation and duration of hospital stay, these four drugs had little or no impact on hospitalized COVID-19 patients. $^{18}$ The Solidarity trial also combined the results of three other remdesivir studies ${ }^{20-22}$ and performed a mortality metaanalysis. The 4 studies on remdesivir were consistent in terms of the dosage, route and frequency of drug use. The combined results showed that remdesivir had no definite effect on mortality (ratio of death rates 0.95 , 95\% confidence interval $0.81-1.11, \mathrm{p}=0.50) .{ }^{18}$ The final report of the NIH remdesivir study (Adaptive COVID-19 Treatment Trial, ACTT-1) indicated that the drug reduced the time to recovery among COVID-19 patients from 15 days to 10 days, but there was no significant mortality benefit. ${ }^{23}$ David J Sher's review assessed remdesivir and hydroxychloroquine in detail. ${ }^{24}$ However, considering that remdesivir does not cause death and serious adverse events by itself, it is still the recommended evidence-based drug treatment. ${ }^{24}$ Obviously, the use of antiviral drugs alone 


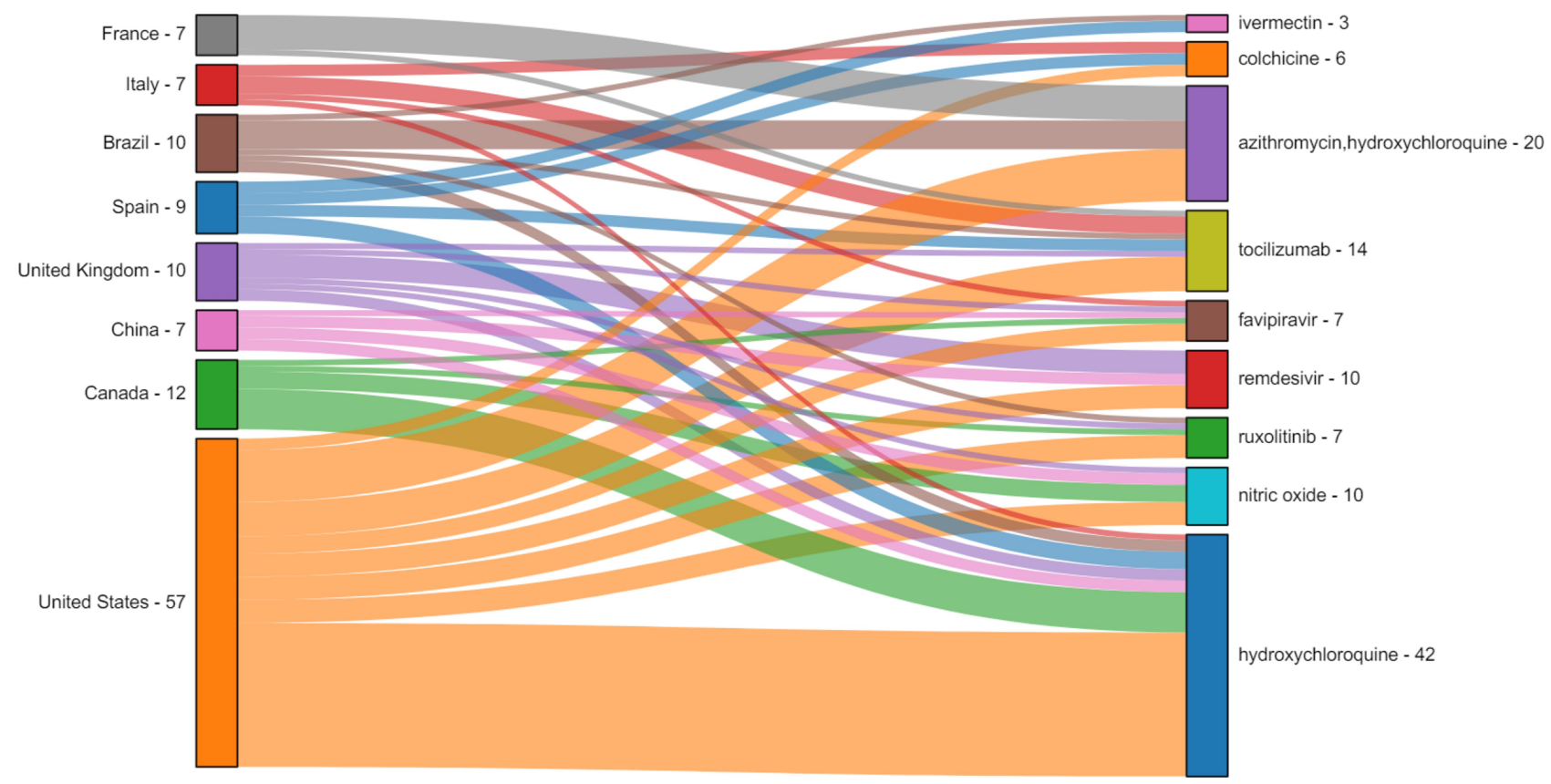

Figure 6 Sankey diagram from countries to drugs. To avoid creating an excessively complex Sankey diagram and increase readability, we controlled the number of drug and country nodes. We first selected the top 9 drug nodes based on the number of trials (with counts greater than 9 ) and then filtered out the main countries contributing to the investigation of these drugs (a total of 8 national nodes with counts greater than 6 ). The filtering threshold was manually determined according to the line density of the Sankey diagram. The Sankey diagram was used to display the flow of the number of trials from one country to a drug; in other words, it is a flow diagram in which the width of arrows is proportional to the degree of flow. The numbers after the country and drug names indicate the total number of outflow and inflow trials, respectively. For example, "United States - 57" means that a total of 57 trials from the United States flowed to different drug nodes on the right side of the figure. "Hydroxychloroquine-42" indicates that a total of 42 hydroxychloroquine trials flowed in from the country nodes on the left. Note that since the remaining nodes are filtered out, these numbers only represent the statistical association between the nodes displayed in the current graph.

may not be enough to reduce mortality among COVID-19 patients. To date, the only group of drugs that has been proven effective for severe and critical COVID-19 patients is corticosteroids. ${ }^{16,24-26}$ Therefore, it is necessary to combine other immunomodulatory drugs and other therapies to improve the prognosis of patients.

Although the results of the Solidarity trial may have set back the search for suitable antiviral drugs, some potentially promising drugs remain. Kumari et al described some of the most promising current drugs in a review of the updated pipeline for pharmacological therapies. ${ }^{27}$ Some new directions include immunomodulators and biologics, traditional Chinese medicines and natural products and herbal medicines. The immunomodulators and biologics mainly consist of convalescent plasma, antibody-based therapy, cell-based therapy (NK cells and mesenchymal stem cells) and other therapies. ${ }^{27}$ Mesenchymal stem cells, a type of plasma immunotherapy, have shown good results in the treatment of COVID-19 patients. ${ }^{28}$ In patients with severe COVID-19, excessive inflammatory cytokine storms and high oxidative stress are mutually promotive and aggravate the deterioration of the disease. ${ }^{29}$ A drug called tocilizumab, a monoclonal antibody, can effectively antagonize IL-6 receptors, ${ }^{30}$ thereby interfering with the inflammatory cascade in patients with severe COVID-19; ${ }^{31}$ some small studies on tocilizumab have reported encouraging effects. ${ }^{32-36}$ The virus-targeting effect of the REGN-COV2 investigational antibody cocktail has been confirmed for SARS-CoV-2, ${ }^{37,38}$ and its Phase 3 trial has been carried out by the RECOVERY trial in the UK. Colchicine can interfere with multiple inflammatory pathways, thus exerting anti-inflammatory and antiviral effects in the treatment of COVID-19. ${ }^{39,40}$ Additionally, melatonin has significant antioxidant and anti-inflammatory properties. ${ }^{41}$ A recently approved study (EudraCT: 2020-001808-42) aims to evaluate its dosage and efficacy in the treatment of COVID-19. ${ }^{42}$ A novel treatment using a combination of methylene blue, vitamin $\mathrm{C}$ and $\mathrm{N}$-acetyl cysteine was used in a Phase 1 trial and achieved good therapeutic effects. ${ }^{43} \mathrm{An}$ epidemiological survey showed that low vitamin D status is associated with an increased risk of COVID-19 infection and hospitalization. ${ }^{44}$ Carlos et al proposed a clinical trial design proposal for vitamin D to prevent COVID-19. ${ }^{45}$

The COVID-19 pandemic has restricted the working methods of many researchers. Therefore, establishing a workflow that can adapt to the pandemic can facilitate more efficient research. ${ }^{46}$ However, four major problems 
remain in the design of clinical trials: the overall trial design (random and sufficient efficacy, ${ }^{47}$ the inclusion of sex as a variable ${ }^{48}$ ), dose optimization, the lack of clear treatment timing window and the selection of appropriate outcome indicators. ${ }^{27}$ For clinical trials on COVID-19 vaccines, Ayoub suggests that multiple doses of Bacillus Calmette-Guérin (BCG) vaccine should be considered a breakthrough in vaccine discovery and could lead to safer results. ${ }^{49}$ For parallel implementations of the same intervention trial, Mathioudakis et al suggest that in terms of methodology, a meta-analysis of the interim data should be analyzed and its key results evaluated by the organization to shorten the time from identification as an effective drug to clinical application. ${ }^{50}$ In terms of research ethics, the research design of COVID-19 trials should not involve reduced ethical review standards. ${ }^{51,52}$

There are several shortcomings in this study. First, we included only the results retrieved from ClinialTrials.gov. However, since we did not perform a search of the many other clinical trial registration websites, our included studies may only represent a small fraction of all studies conducted worldwide. Second, we only extracted some of the more important clinical trial feature variables. The registration information of the clinical trials contains a wealth of content, so some potentially important characteristic information may have been missed. Finally, our Sankey diagram does not include a sample size edge label between the drug and country node; subsequent research will provide more valuable information if it can be expanded to visualize the relationship between countries, drugs, and sample sizes.

\section{Conclusion}

The designs of COVID-19 clinical drug trials have greatly improved in terms of the implementation of randomization and, particularly, blinding methods. Additionally, the number of drug types has greatly increased, and hundreds of drugs have now been assessed for efficacy screening. The emergence of large-sample registration trials is expected to address the uncertainty surrounding the current clinical efficacy of some drugs. The distribution of many suitable drug trials across different countries around the world has promoted the possibility of multinational collaboration. Although many trials have been registered and half of the trials are actively recruiting, much work remains in the search for evidence on possible therapeutic drugs, especially for the one-third of overall trials that involve small sample sizes.

\section{Abbreviations}

COVID-19, coronavirus disease 2019; NIH, National Institutes of Health; WHO, World Health Organization; RECOVERY, Randomised Evaluation of COVID-19 Therapy; ACTT-1, Adaptive COVID-19 Treatment Trial; BCG, Bacillus Calmette-Guérin.

\section{Data Sharing Statement}

All data can be obtained from the publicly accessible ClinialTrials.gov (https://clinicaltrials.gov).

\section{Funding}

This study was supported by the National Science and Technology Key Project (No. 2017ZX10106001003), Comprehensive Research on COVID-19 Prevention for Emergency Scientific Research Project (No. GSEBMKT2020YJ03), and Science and Technology Project of Lanzhou City (No. 2020SHFZ0041).

\section{Disclosure}

All authors declare no potential conflicts of interest in this work.

\section{References}

1. Jiang F, Deng L, Zhang L, Cai Y, Cheung CW, Xia Z. Review of the clinical characteristics of coronavirus disease 2019 (COVID-19). $J$ Gen Intern Med. 2020;35(5):1545-1549. doi:10.1007/s11606-02005762-w

2. Jean -S-S, Lee P-I, Hsueh P-R. Treatment options for COVID-19: the reality and challenges. $J$ Microbiol Immunol Infect. 2020;53 (3):436-443. doi:10.1016/j.jmii.2020.03.034

3. Wang B, Lai J, Yan X, Jin F, Yao C. Implications of the lack of a unified research project framework: an investigation into the registration of clinical trials of COVID-19. Curr Med Res Opin. 2020;36 (7):1131-1135. doi:10.1080/03007995.2020.1771294

4. Wang B, Lai J, Yan X, Jin F, Yao C. Exploring the characteristics, global distribution and reasons for retraction of published articles involving human research participants: a literature survey. Eur J Intern Med. 2020;78:145-146. doi:10.1016/j.ejim.2020.03.016

5. Mehra MR, Ruschitzka F, Patel AN. Retraction-Hydroxychloroquine or chloroquine with or without a macrolide for treatment of COVID-19: a multinational registry analysis. Lancet. 2020;395 (10240):1820. doi:10.1016/S0140-6736(20)31324-6

6. Mehra MR, Desai SS, Kuy S, Henry TD, Patel AN. Retraction: cardiovascular disease, drug therapy, and mortality in Covid-19. N Engl J Med. N Engl J Med. 2020;382(26):2582. doi:10.1056/ NEJMoa2007621

7. Zhang X, Yu J, Pan L-Y, Jiang H-Y. ACEI/ARB use and risk of infection or severity or mortality of COVID-19: a systematic review and meta-analysis. Pharmacol Res. 2020;158:104927. doi:10.1016/j. phrs.2020.104927

8. Meyerowitz EA, Vannier AGL, Friesen MGN, et al. Rethinking the role of hydroxychloroquine in the treatment of COVID-19. FASEB J. 2020;34(5):6027-6037.

9. Touret F, de Lamballerie X. Of chloroquine and COVID-19. Antiviral Res. 2020;177:104762. doi:10.1016/j.antiviral.2020.104762 
10. Rosa SGV, Santos WC. Clinical trials on drug repositioning for COVID-19 treatment. Rev Panam Salud Publica. 2020;44:e40. doi:10.26633/RPSP.2020.40

11. Nasrallah AA, Farran SH, Nasrallah ZA, et al. A large number of COVID-19 interventional clinical trials were registered soon after the pandemic onset: a descriptive analysis. $J$ Clin Epidemiol. 2020;125:170-178. doi:10.1016/j.jclinepi.2020.06.005

12. Boserup B, McKenney M, Elkbuli A. An overview of current COVID-19 clinical trials and ethical considerations editorial. Ann Med Surg (Lond). 2020;58:84-86. doi:10.1016/j.amsu.2020.08.041

13. Idda ML, Soru D, Floris M. Overview of the first 6 months of clinical trials for COVID-19 pharmacotherapy: the most studied drugs. Front Public Health. 2020;8:497. doi:10.3389/fpubh.2020.00497

14. Karlsen APH, Wiberg S, Laigaard J, Pedersen C, Rokamp KZ, Mathiesen O. A systematic review of trial registry entries for randomized clinical trials investigating COVID-19 medical prevention and treatment. PLoS One. 2020;15(8):e237903. doi:10.1371/journal.pone.0237903

15. Vanden Eynde JJ. COVID-19: a brief overview of the discovery clinical trial. Pharmaceuticals (Basel). 2020;13:4.

16. Siemieniuk RA, Bartoszko JJ, Ge L, et al. Drug treatments for covid-19: living systematic review and network meta-analysis. BMJ. 2020;370:m2980. doi:10.1136/bmj.m2980

17. Horby P, Mafham M, Linsell L, et al. Effect of hydroxychloroquine in hospitalized patients with Covid-19. N Engl J Med. 2020.

18. Pan H, Peto R, Abdool Karim Q, et al. Repurposed antiviral drugs for COVID-19; interim WHO SOLIDARITY trial results. medRxiv. 2020;2020(2010):2015.20209817.

19. Boulware DR, Pullen MF, Bangdiwala AS, et al. A randomized trial of hydroxychloroquine as postexposure prophylaxis for Covid-19. $N$ Engl J Med. 2020;383(6):517-525. doi:10.1056/NEJMoa2016638

20. McMahon JH, Udy A, Peleg AY. Remdesivir for the treatment of Covid-19 - preliminary report. $N$ Engl J Med. 2020;383 (10):992-993.

21. Spinner CD, Gottlieb RL, Criner GJ, et al. Effect of remdesivir vs standard care on clinical status at 11 days in patients with moderate COVID-19: a randomized clinical trial. JAMA. 2020;324 (11):1048-1057. doi:10.1001/jama.2020.16349

22. Wang Y, Zhang D, Du G, et al. Remdesivir in adults with severe COVID-19: a randomised, double-blind, placebo-controlled, multicentre trial. Lancet. 2020;395(10236):1569-1578. doi:10.1016/ S0140-6736(20)31022-9

23. Beigel JH, Tomashek KM, Dodd LE, et al. Remdesivir for the treatment of Covid-19 - final report. N Engl J Med. 2020.

24. Sher DJ. An ounce of prevention and a pound of cure: randomized clinical trials of therapeutics against COVID-19 and an assessment of personal protective equipment and distancing. Int J Radiat Oncol Biol Phys. 2020;108(2):333-336. doi:10.1016/j.ijrobp.2020.08.001

25. Sharun K, Tiwari R, Dhama J, Dhama K. Dexamethasone to combat cytokine storm in COVID-19: clinical trials and preliminary evidence. Int J Surg. 2020;82:179-181. doi:10.1016/j.ijsu.2020.08.038

26. Brotherton H, Usuf E, Nadjm B, et al. Dexamethasone for COVID-19: data needed from randomised clinical trials in Africa. Lancet Glob Health. 2020;8(9):e1125-e1126. doi:10.1016/S2214109X(20)30318-1

27. Kumari P, Rawat K, Saha L. Pipeline pharmacological therapies in clinical trial for COVID-19 pandemic: a recent update. Curr Pharmacol Rep. 2020;1-13.

28. Zhang Y, Ding J, Ren S, et al. Intravenous infusion of human umbilical cord Wharton's jelly-derived mesenchymal stem cells as a potential treatment for patients with COVID-19 pneumonia. Stem Cell Res Ther. 2020;11(1):207. doi:10.1186/s13287-020-01725-4

29. Hamidi Alamdari D, Bagheri Moghaddam A, Amini S, Hamidi Alamdari A, Damsaz M, Yarahmadi A. The application of a reduced dye used in orthopedics as a novel treatment against Coronavirus (COVID-19): a suggested therapeutic protocol. Arch Bone Jt Surg. 2020;8(Suppl1):291-294.
30. Fitzgerald JC, Weiss SL, Maude SL, et al. Cytokine release syndrome after chimeric antigen Receptor T cell therapy for acute lymphoblastic leukemia. Crit Care Med. 2017;45(2):e124-e131. doi:10.1097/ CCM.0000000000002053

31. Abrams-Downey A, Saabiye J, Vidaurrazaga M. Investigational therapies for the treatment of COVID-19: updates from ongoing clinical trials. Eur Urol Focus. 2020;6(5):1028-1031. doi:10.1016/j. euf.2020.05.019

32. Dastan F, Saffaei A, Haseli S, et al. Promising effects of tocilizumab in COVID-19: a non-controlled, prospective clinical trial. Int Immunopharmacol. 2020;88:106869. doi:10.1016/j.intimp.202 0.106869

33. Xu X, Han M, Li T, et al. Effective treatment of severe COVID-19 patients with tocilizumab. Proc Natl Acad Sci US A. 2020;117 (20):10970-10975. doi:10.1073/pnas.2005615117

34. Luo P, Liu Y, Qiu L, Liu X, Liu D, Li J. Tocilizumab treatment in COVID-19: a single center experience. J Med Virol. 2020;92 (7):814-818. doi:10.1002/jmv.25801

35. Guaraldi G, Meschiari M, Cozzi-Lepri A, et al. Tocilizumab in patients with severe COVID-19: a retrospective cohort study. Lancet Rheumatol. 2020;2(8):e474-e484. doi:10.1016/S26659913(20)30173-9

36. Alattar R, Ibrahim TBH, Shaar $\mathrm{SH}$, et al. Tocilizumab for the treatment of severe coronavirus disease 2019. J Med Virol. 2020;92 (10):2042-2049. doi:10.1002/jmv.25964

37. Hansen J, Baum A, Pascal KE, et al. Studies in humanized mice and convalescent humans yield a SARS-CoV-2 antibody cocktail. Science. 2020;369(6506):1010-1014.

38. Baum A, Fulton BO, Wloga E, et al. Antibody cocktail to SARS-CoV-2 spike protein prevents rapid mutational escape seen with individual antibodies. Science. 2020;369(6506):1014-1018.

39. Schlesinger N, Firestein BL, Brunetti L. Colchicine in COVID-19: an old drug, new use. Curr Pharmacol Rep. 2020;1-9.

40. Siebenand VS. COVID-19: colchicine in clinical trials. Pharmazeutische Zeitung. 2020;165(21):19.

41. Feitosa EL, Júnior F, Nery Neto JAO, et al. COVID-19: rational discovery of the therapeutic potential of Melatonin as a SARS-CoV-2 main protease inhibitor. Int J Med Sci. 2020;17 (14):2133-2146. doi:10.7150/ijms.48053

42. Acuña-Castroviejo D, Escames G, Figueira JC, de la Oliva P, Borobia AM, Acuña-Fernández C. Clinical trial to test the efficacy of melatonin in COVID-19. J Pineal Res. 2020;69(3):e12683. doi:10.1111/jpi.12683

43. Alamdari DH, Moghaddam AB, Amini S, et al. Application of methylene blue -vitamin $\mathrm{C}$-N-acetyl cysteine for treatment of critically ill COVID-19 patients, report of a phase-I clinical trial. Eur J Pharmacol. 2020;885:173494. doi:10.1016/j.ejphar.2020.173494

44. Merzon E, Tworowski D, Gorohovski A, et al. Low plasma 25(OH) vitamin D level is associated with increased risk of COVID-19 infection: an Israeli population-based study. FEBS J. 2020;287 (17):3693-3702. doi:10.1111/febs. 15495

45. Camargo CA Jr, Martineau AR. Vitamin D to prevent COVID-19: recommendations for the design of clinical trials. FEBS J. 2020;287 (17):3689-3692. doi:10.1111/febs.15534

46. Emamaullee J, Bowdish M, Yan PY, Rodina V, Sher LS. Rapid adaptation of a surgical research unit to conduct clinical trials during the COVID-19 pandemic. J Surg Res. 2020;256:76-82. doi:10.1016/ j.jss.2020.06.049

47. Yao C. Clinical trial in China: the status and challenge of data management and statistical analysis. J Evid Based Med. 2018;11 (1):3-6. doi:10.1111/jebm.12295

48. Bischof E, Wolfe J, Klein SL. Clinical trials for COVID-19 should include sex as a variable. J Clin Invest. 2020;130(7):3350-3352. doi:10.1172/JCI139306

49. Ayoub BM. COVID-19 vaccination clinical trials should consider multiple doses of BCG. Pharmazie. 2020;75(4):159. 
50. Mathioudakis AG, Fally M, Hashad R, Knight S, Felton T, Vestbo J. COVID-19 clinical trials: unraveling a methodological gordian knot. Am J Respir Crit Care Med. 2020;202(5):635-637. doi:10.1164/ rccm.202005-1942ED

51. Han Z, Wang J, Zhang K, Tang Q. The ethics of COVID-19 clinical trials: new considerations in a controversial area. Integr Med Res. 2020;9(3):100425. doi:10.1016/j.imr.2020.100425
52. Singh AG, Chaturvedi P. Clinical trials during COVID-19. Head Neck. 2020;42(7):1516-1518. doi:10.1002/hed.26223

\section{Publish your work in this journal}

Drug Design, Development and Therapy is an international, peerreviewed open-access journal that spans the spectrum of drug design and development through to clinical applications. Clinical outcomes, patient safety, and programs for the development and effective, safe, and sustained use of medicines are a feature of the journal, which has also been accepted for indexing on PubMed Central. The manuscript management system is completely online and includes a very quick and fair peer-review system, which is all easy to use. Visit http://www. dovepress.com/testimonials.php to read real quotes from published authors. 